\title{
An adaptive Model of Achilles Tendon Mechanical Properties during Adolescence: Effect of Sex
}

\author{
G. Chalatzoglidis ${ }^{1}$, A. J. Blazevich ${ }^{2}$, F. Arabatzi ${ }^{1}$ \\ 1 Laboratory of Neuromechanics, Department of Physical Education and Sport Science, \\ Aristotle University of Thessaloniki, Serres, Greece \\ 2 Centre for Exercise and Sports Science Research, School of Medical and Health Sciences, \\ Edith Cowan University, Joondalup, Australia
}

\author{
CORRESPONDING AUTHOR: \\ Georgios Chalatzoglidis \\ Laboratory of Neuromechanics \\ Department of Physical Education and \\ Sport Science \\ Aristotle University of Thessaloniki \\ 62110 Serres, Greece \\ E-mail: gchalatzo@phed-sr.auth.gr \\ DOI: \\ 10.32098/mltj.02.2021.11
}

LEVEL OF EVIDENCE: $1 \mathrm{~B}$

\begin{abstract}
SUMMARY
Background. This longitudinal study aimed to quantify the relative effects of body mass, peak muscle force, maturation and sex on Achilles tendon (AT) mechanical properties and to examine the external factors that trigger mechanical changes, or intrinsic tendon adaptations during adolescence.

Methods. We measured AT mechanical properties and dimensions during pre-pubertal and adolescent growth in 41 participants ( 20 boys and 21 girls). Participants were tested over 18 months; longitudinal changes were examined through linear mixed modelling.

Results. Sex and maturation were found to be the major factors influencing AT mechanical changes. Their effects were largely exerted through increases in muscle force, which imposed greater stress on AT and strongly predicted changes in stiffness and Young's modulus in boys and girls, while strain was consistent.

Conclusions. The more rapid increase in stiffness before the age of peak height velocity in boys was associated with an increase in force at that time, which may have evoked the molecular signaling required for adaptations to internal tendon structure leading a different adaptive response between sexes. The present data are suggestive of an adaptive model in which increases in muscle force production impose greater mechanical loading, on the tendon to trigger increases in stiffness as children mature through adolescence.
\end{abstract}

KEY WORDS

Tendon stiffness; stress; strain; adaptation; sex; adolescence.

\section{BACKGROUND}

The human Achilles tendon (AT) is a spring-like, load-bearing structure that transmits muscular forces directly to the calcaneum of the foot, playing an essential role during daily tasks such as walking, running and jumping. AT would theoretically need to adapt rapidly to changes in body mass and strength, such as during periods of body growth and maturation. In the present study we investigated the longitudinal changes in the properties of the Achilles tendon in adolescent girls and boys during a time period around growth-spurt. Cross-sectional data from human studies suggests that the timing and rate of maturation during growth from childhood varies greatly between individuals (1), with a rapid period of growth associated with the peak height velocity (PHV) being clearly observed in the pubertal phase. However, the limited existing data obtained by cross-sectional analyses suggests that significant increases in both tendon stiffness and Young's modulus occur with maturation to adulthood $(2,3)$, with a rapid increase in stiffness at or around PHV resulting partly from increases in cross-sectional area (2, 3 ), potentially alongside a slowing in the rate of increase in tendon length (4). Collectively, these data provide an uncomplicated picture of the growth characteristics of important, energy-storing tendons such at the human AT. However, it is not yet known whether cross-sectional analyses accurately describe the longitudinal adaptive process.

The mechanical properties of the Achilles tendon also have been shown to differ based on both sex (5) and growth 
rate (6). Although asynchronous growth rates may affect stress, strain, and stiffness of AT, especially in the PHV period, growth itself might not be a major factor influencing muscle-tendon unit (MTU) strain, and thus mechanical change. Indeed, the cross-sectional data of Mogi, Torii (2) indicated that the calf-Achilles MTU length increases concurrently with the longitudinal growth of bones even in the period of rapid adolescent growth. In the only longitudinal study thus far completed, Neugebauer and Hawkins (6) did not detect changes in peak AT strain, stiffness or modulus over a 6-month period, and no statistical relationships with sex, growth rate or physical activity levels were observed in a sample of 10-12 years old girls and 12-14 years old boys. However, it is not known whether changes, if present, can be reliably detected over a 6-month period using current testing methods. Furthermore, we do not perfectly know whether boys and girls adapt similarly, whether their changes occur at the same rate relative to PHV, or whether changes are similar in low or high force levels. By tracking changes over a longer time period, age- and maturation-related changes could be clarified.

Body mass increases significantly around PHV (1), which results in rapid increases in muscle force and consequently a greater mechanical load on tendons (7). These changes have been associated with increased stiffness $(\mathrm{k})$ and Young's modulus (E) $(3,6)$, but it is not clear yet whether body mass, strength or both are explanatory factors of tendon adaptation. Furthermore, increases in Young's modulus, are related to either repeated stress (8) or high strain magnitudes (9). Whereas the strain at tendon failure is more or less constant during maturation the stress to tendon failure is dependent on the tendon's material properties. Peak AT stress increased over a 6-month period due to a decrease in AT CSA in the study of Neugebauer and Hawkins (6) whereas greater (by percentage) increases in tendon stiffness and cross-sectional area than both muscle size and strength were observed over 2 years in the patellar tendon of adolescent volleyball players, suggesting that growth was associated with a reduced peak tendon stress (10). However, it is not certain whether the peri-adolescent period is associated with significant increases in stress or strain that might predispose to adaptation and or injury (11). Therefore, the examinations of AT properties over a similar period might allow for the rates of changes in mechanical variables to be more clearly established and for associations with variables such as sex, maturation, body mass and peak muscular strength to be explored. The current literature remains limited primarily to cross-sectional studies, thus a more complete picture of tendon adaptation could then be feasibly drawn from the simultaneous tracking of children at different ages and levels of maturation at the start of the 1.5 -year period.
Complicating matters is that tendon exhibits highly non-linear axial force-displacement behavior at low force (low strain) levels and linear behavior at higher forces (higher strain). Little is known about the relative changes in the low-force region of the force-length (stress-strain) relation in AT or other energy-storing tendons, which is problematic given that in vivo loading of the human AT typically lies within the low-strain region in most activities of daily living. Therefore, it is of great interest to specifically examine age-related changes in tendon properties, in both sexes, in the low-to-moderate force level region.

Given the above, the purpose of the present longitudinal study was to quantify the relationships between body mass, peak muscle force (strength), maturation and sex with AT mechanical properties including stiffness, Young's modulus, stress and strain. It was hypothesized that changes in both body mass and strength would be strongly associated with longitudinal changes in AT mechanical properties in both low- and high-force regions, but that these would be underpinned by the maturation process in a sex-dependent manner which may varies at different force levels during the adolescent growth spurt.

\section{MATERIALS AND METHODS}

Fifty-seven prepubertal children volunteered for the study, while forty-one participants (20 boys: $12.6 \pm 0.4$ years, 48.7 $\pm 7.2 \mathrm{~kg}$, and $157.6 \pm 5.1 \mathrm{~cm} ; 21$ girls: $10.6 \pm 0.5$ years, 36.6 $\pm 6.8 \mathrm{~kg}$, and $146.4 \pm 8.1 \mathrm{~cm}$ ) were found to be close to the age of PHV, 16 participants were excluded since no PHV was observed. The predicted age of PHV was based on the equation of Mirwald, Baxter-Jones (12). The observed age of PHV that was closer to the best predicted age of PHV was accepted as the age of peak height velocity (APHV) in each participant. In order to determine the degree of reliability of the above assumption, the intraclass correlation coefficient (ICC) between the two ages (best predicted and APHV) was calculated. The ICC rate for boys was 0.97 (p $<0.001)$ and for girls $0.77(\mathrm{p}<0.001)$. The acceptable limit of the coefficient is $>0.75$ (13), thus the best predicted age of PHV did not differ significantly from the approximate APHV defined in the present study.

Complete anthropometric characteristics for each sex at each testing time point are shown in table I. The age ranges represent one standard deviation around the average age at which peak height velocity (PHV) occurs for boys and girls, respectively. Finally, the maturation progress was classified into 4 stages: 12,6 mo before, and then 0 , and 6 months after, the age of PHV (corresponding to $1^{\text {st }}, 2^{\text {nd }}$, $3^{\text {rd }}$ and $4^{\text {th }}$ time point of measurement, respectively). The difference of 6 mo in the predicted PHV was accepted 
Table I. Anthropometric characteristics of participants per stage of maturity (months from predicted age of PHV): age (years), body mass $(\mathrm{kg})$, height $(\mathrm{cm})$. A significant change in height $\left.{ }^{*} 0.001<p<0.01\right)$ was observed in the interval between 1 year before and the predicted age of PHV.

\begin{tabular}{lllccc}
\hline Maturity stage & & $\mathbf{- 1 2}$ & $\mathbf{- 6}$ & $\mathbf{0}$ & $\mathbf{+ 6}$ \\
\hline Boys $(\mathrm{N}=21)$ & Age & $12.6 \pm 0.4$ & $13.4 \pm 0.4$ & $13.9 \pm 0.4$ & $15.2 \pm 0.7$ \\
\cline { 2 - 5 } & Body mass & $48.7 \pm 7.2$ & $53.2 \pm 7.3$ & $59.1 \pm 7.2$ & $61.3 \pm 8.3$ \\
\cline { 2 - 5 } & Height & $157.6 \pm 5.1$ & $162.1 \pm 7.2$ & $169 \pm 7.5^{*}$ & $173.2 \pm 10.7$ \\
\hline \multirow{2}{*}{ Girls $(\mathrm{N}=20)$} & Age & $10.6 \pm 0.5$ & $11.5 \pm 0.5$ & $12 \pm 0.5$ & $13.05 \pm 0.5$ \\
\cline { 2 - 5 } & Body mass & $36.6 \pm 6.8$ & $41.6 \pm 7.9$ & $46.8 \pm 8$ & $50.0 \pm 8.5$ \\
\cline { 2 - 5 } & Height & $146.4 \pm 8.1$ & $151.4 \pm 7.9$ & $158.1 \pm 6.9^{*}$ & $162.2 \pm 4.0$ \\
\hline
\end{tabular}

within the acceptable range ( $<1$ year) as recommended by Mirwald, Baxter-Jones (12). All participants were recruited from public schools and were healthy, without any disability or leg injury. All students and parents provided informed consent. Research protocol and measurement techniques were approved by Local Ethics Research Committee (ERC003/2020). The study was conducted in accordance with the ethical guidelines and recommendations for the clinical and field science research (14).

\section{Experimental design - measurements}

Participants were tested in a random order over 18 months by performing the same measurements under similar conditions at 6-month intervals. Anthropometric data including standing height, sitting height, leg length, body mass and age were recorded at each testing session (every six months, 4 time points) and used to ensure the validity of procedure and calculate the maturity offset. Subsequently, the participants laid prone on the dynamometer bench (Cybex Humac Norm, CSMI, MA, USA) for strength measurements (figure 1). The instrument and measurement accuracy were $\pm 0.5 \%$ full-scale maximum. The foot was positioned perpendicular to the tibia (ankle in the neutral position, knee fully extended) and placed in the dynamometer's foot plate with right ankle in the neutral position $\left(0^{\circ}\right.$ : foot was perpendicular to the longitudinal axis of the tibia). The foot was tightly secured with straps to the footplate and the hips were tightly strapped to the seat. The rotation axis of the ankle joint was carefully aligned to be parallel to the axis of the lever arm of the dynamometer and passing through the midpoint of the line connecting both malleoli, as described by De Monte, Arampatzis (15). B-mode ultrasonography (US) (SSD-3500, Aloka, Tokyo, Japan) using a 60-mm electronic linear-array probe (7.5 MHz wave frequency) was used to measure the displacement of the distal myotendinous junction (MTJ) of the medial gastrocnemius (MG) during ramped, maximal isometric plantar flexions. The probe was placed longitudi- nally at the position specified by a marker attached on the skin surface. An echo-absorptive marker was attached to monitor possible motion of the probe on the skin during the measurement. Analog signals from the dynamometer were amplified and synchronized with data from the ultrasound system using a DA 100 B amplifier (Biopac Systems. Inc., Goleta, CA, common mode rejection ratio $>90 \mathrm{db}$, bandwidth $=0.05-500 \mathrm{~Hz}$ ). Torque data were recorded by the dynamometer at a sampling frequency of $100 \mathrm{~Hz}$.

\section{Familiarization}

Prior to testing, each participant performed warm-up exercise consisting of 3-5 sub-maximal isometric plantar flex-

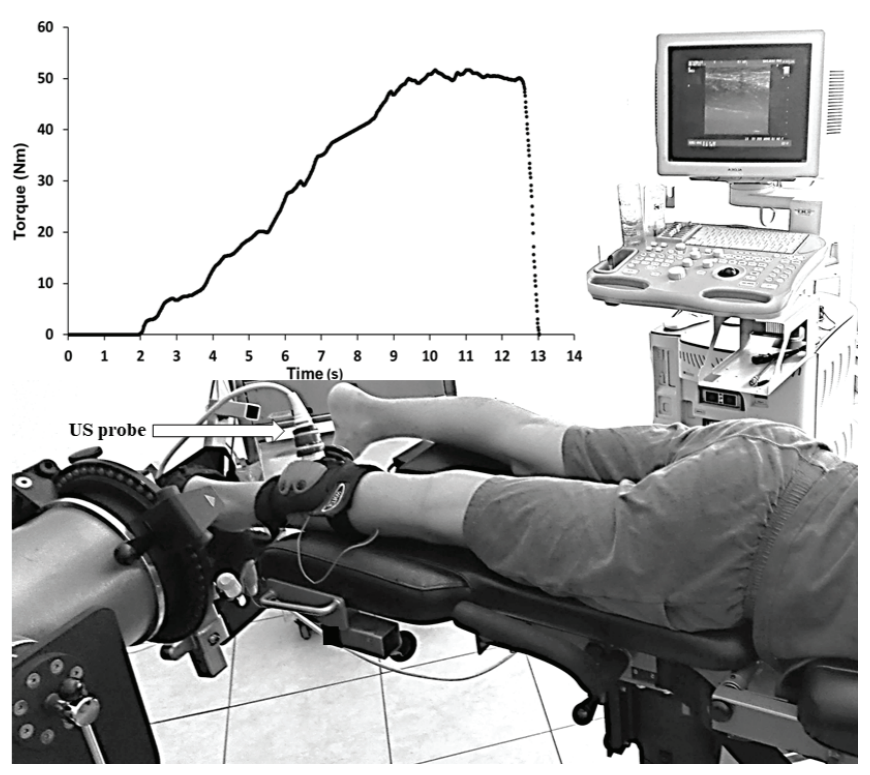

Figure 1. Experimental setup, showing the ultrasound probe fixed to the muscle-tendon junction during ankle joint moment data collection. Upper left corner: torque-time trace (raw data) showing linear torque $(\mathrm{Nm})$ increase over time (s). 
ion contractions to pre-condition the tendon. Thereafter, three ramped isometric plantar flexions (IPF) with maximal effort were done to familiarize the participants with the IPF required for testing. A 5 -min passive rest was given between the familiarization phase and the testing protocol to minimize fatigue.

\section{Torque measurement during plantar flexion}

Upon the instructor's request, participants performed two 10 -s isometric ramp plantar flexion contractions increasing from $0 \%$ to $100 \%$ of the maximum torque at $20 \%$ of maximum torque every $2 \mathrm{~s}$ and holding maximally for $5 \mathrm{~s}$ (30-s inter-trial rest). In addition to continuous verbal encouragement, the participant was provided visual feedback of the torque signal on screen to allow execution of the effort as accurately as possible. Torque values were obtained from the best effort in which the ramp was properly achieved and torque-time relations were linear in the ramp period without energy loss (resulting from notable decreases in torque) in the test.

\section{Measurement of tendon elongation}

Ultrasound images were digitally recorded at a sampling frequency of $25 \mathrm{~Hz}$. Max TRAQ software (Max Traq Lite version 2.09, Innovision Systems, Inc. Columbiaville, Michigan. U.S.A) was used to digitize the spatial location of the MTJ in order to generate MTJ position-time data from the ultrasound video. 25 position points (1 position point every $0.4 \mathrm{~s}$ ) were recorded for each 10-s ramp contraction. AcqKnowledge Acquisition \& Analysis Software (BIOPAC Systems, Inc., USA) was used to synchronize the torque signal and ultrasound imaging data. MG MTJ displacement was assumed to represent the change in the length of the AT. An example of Torque-elongation relationship obtained during ramped plantarflexion contractions for an adolescent girl (12 years old) during age of peak height velocity is shown in figure 2 .

As reported elsewhere (16), both a shift of the foot from the initial position and a change in ankle joint angle were observed during the measurements. The detection of possible heel movement during contraction was provided by capturing high-speed digital video (JVC 9800, frame rate = $120 \mathrm{~Hz}$ ) with a high-speed shutter to record plantar flexion motion. Five reflectors $(2.5$-mm radius) were placed on the $5^{\text {th }}$ metatarsal, lateral malleolus, and lower extremity of the heel and two markers were placed on the foot plate. The video camera was placed opposite the ankle in the frontal plane at the middle of the dynamometer's foot plate. Despite foot stabilization with the elastic straps and bandag-

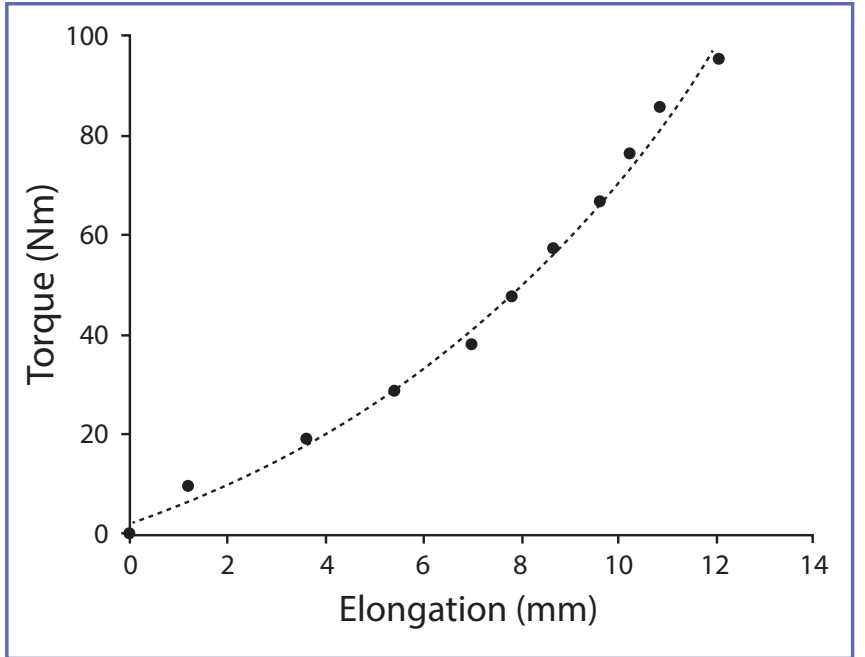

Figure 2. Example of Torque-elongation relationship obtained during ramped plantarflexion contractions for an adolescent girl (12 years old) during age of peak height velocity.

es, displacement of the ankle joint was recorded during contraction, resulting in loss of alignment with the dynamometer rotation axis. To accurately calculate the angle of rotation in two dimensions, the positions of the reflectors were recorded during the ramp isometric plantar flexion contraction and coordinate data from high-speed camera were down-sampled to match the ultrasound sampling frequency. The additional MTJ positional change $(\Delta \mathrm{L} ; \mathrm{mm})$ due to ankle rotation was calculated from the angle $\Delta \varphi$ (rad) recorded by the camera and the torque moment arm's length (d; mm) according to the equation: $\Delta \mathrm{L}=\Delta \varphi \times \mathrm{d}$. The additional MTJ displacement was subtracted from recorded elongation $(\Delta \mathrm{L})$ to compensate for the influence of ankle joint rotation.

\section{Moment arm measurement}

The perpendicular distance from the center of rotation to the line of AT action was defined as AT moment arm (MA), which was calculated with the excursion method. Following the execution of the measurement protocol, without changing the participant's position or the position of the ultrasound probe, passive AT behavior assessment was performed with the dynamometer rotating passively between $20^{\circ}$ dorsiflexion and $20^{\circ}$ plantarflexion at a speed of $5 \%$. The instruction was given to the participants to relax their leg muscles during the dynamometer's foot plate movement to minimize muscle activity. Six trials were executed, and data were collected from the rotation in which the MTJ location was best visualized. MTJ position data were collected initially as video 
recordings and Acknowledge software was used to synchronize the MTJ movement and angular position. A $3^{\text {rd }}$-order polynomial was fitted to describe the relationship between angular position and elongation of AT, and the first derivative of the polynomial for angle $0^{\circ}$ was calculated to find the slope of the curve which represents the moment arm. Previous studies have not detected any difference between AT moment arm estimations between rest and MVC using the tendon excursion method over any given foot rotation step (17). This was attributed to the fact that although both elongation $(\Delta \mathrm{L})$ and angular displacement $(\mathrm{d} \varphi)$ were altered during MVC compared with rest at any angle, their ratio, which determines the magnitude of moment arm value, remained constant between contraction conditions.

\section{Calculation of the AT force}

The tendon's force was estimated by the equation: $\mathrm{F}=\mathrm{M} / \mathrm{d}$, where:

- $\mathrm{M}$ is the plantarflexion moment;

- $\mathrm{d}$ the AT MA length.

In this study, the isometric plantar flexion force was calculated at angle $0^{\circ}$. The force resulting from this calculation represents the total force of all the ankle muscles transferred through the AT. In order to estimate the antagonistic moment of tibialis anterior (TA), moment and EMG signals from tibialis anterior were measured during ramped isometric dorsiflexions. The TA, as the major dorsiflexor muscle, was assumed to represent antagonist co-activation during plantarflexion. The EMG signals were recorded using pre-gelled $\mathrm{Ag}-\mathrm{AgCl}$ surface circular electrodes with $10 \mathrm{~mm}$ diameter placed $20 \mathrm{~mm}$ apart located at $1 / 3$ between the tip of fibula and the tip of medial malleolus and placed parallel to the TA fibers, in conformity with European recommendations for the surface EMG assessment of muscles. Reference electrodes were placed around the ankle. EMG signals captured by each of the electrode pairs over tibialis anterior were smoothed using a digital low-pass filter. The maximal plantar flexor moment was corrected by the antagonistic moment values. The plantar flexor moment was calculated by finding the sum of the resultant joint moment and antagonistic moment.

\section{Tendon stiffness calculation}

The stiffness $\mathrm{k}(\mathrm{N} / \mathrm{mm})$ was defined as the slope of the linear portion of force $(\mathrm{F})$ - elongation $(\Delta \mathrm{L})$ relation. The area of the linear trend $(\mathrm{F}-\Delta \mathrm{L})$ was accepted as the area between $60 \%$ and $90 \%$ of the peak force $(3,17)$. In order to plot the F- $\Delta \mathrm{L}$ relation, data from the force-time calcu- lation and data from the MTJ position-time relation were combined in each ramp isometric plantar flexion for all measurements. Subsequently, muscle force $(\mathrm{N})$ and the MTJ length change $(\mathrm{mm})$ data were synchronized and the slope of the F- $\Delta \mathrm{L}$ relation was calculated: $\mathrm{k}=\mathrm{dF} / \mathrm{dL}(\mathrm{N} /$ $\mathrm{mm})$. Force-elongation values ranged from $10 \%$ to $40 \%$ and $60 \%$ to $90 \%$ (linear region) of the maximum force. A $1^{\text {st }}$-order polynomial was applied where the slope of the low-force and linear regions of the F- $\Delta \mathrm{L}$ relation was equal to AT stiffness. Stiffness in the $10 \%$ to $40 \%$ region was measured from the length in relaxed muscle at a $0^{\circ}$ joint angle (that is, at the length during standing but with no muscle activity) and that during contraction. The ankle angle was thus tested under identical conditions at each time point and between individuals.

\section{Measurement of AT test length and cross- sectional area $\left(\mathrm{CSA}_{\mathrm{AT}}\right)$}

Slack length is usually assumed to be the length measured either with the joint in its mid-position or when the net joint torque is zero and the resting length can only be defined when the muscle is completely slack. There is currently no method to characterize tendon resting length in vivo, i.e., it cannot be ascertained whether force was zero, hence in our study the 'test' length was measured. Therefore, a standardized joint configuration was set to control tendon length before muscle contraction. With the leg muscles relaxed, the MTJ was located with the ultrasound probe placed on the skin above and parallel to GM. Measurements were performed by sagittal imaging where the tendon boundaries were defined from AT insertion on the calcaneal tuberosity to the GM MTJ. The positions of the MTJ and the insertion of the tendon onto the calcaneal tuberosity were carefully marked on the skin and then the distance measured as a straight line. The vertical distance to the contact surface of the probe from the MTJ was measured by ultrasound. The test length of AT was defined as the hypotenuse of the Pythagorean triangle.

Two main imaging techniques have been used to estimate in vivo $\mathrm{CSA}_{\mathrm{AT}}$ : magnetic resonance imaging and $\mathrm{B}$-mode ultrasound. To measure $\mathrm{CSA}_{\mathrm{AT}}$, a transverse section was visualized using B-mode ultrasound. A special gel patch (gel pad Aquaflex, $2 \times 9 \mathrm{~cm}$ Parker Laboratories, Inc., Fairfield, NJ USA) was used to enhance acoustic conductivity and obtain clearer images where $\mathrm{CSA}_{\mathrm{AT}}$ is smaller and receives the greatest pressure. The minimum CSA $\mathrm{AT}_{\mathrm{AT}}$ was the one accepted for the measurement. The area evaluated as minimum $\mathrm{CSA}_{\mathrm{AT}}$ was the mean value of three recorded measurements by subtracting and replacing the gel patch each time. This ultrasonography technique is widely used to measure AT cross-sectional area (18). However, Bohm, Mersmann (19) showed that 
US measurements were smaller (19\%) than those obtained using MRI method. We therefore ran the analysis with and without the $19 \%$ correction and found the same outcome, so any potential underestimation would not have influenced the results as long as that error was consistent. The reliability of $\mathrm{CSA}_{\mathrm{AT}}$ measurements was investigated in a pilot study of 6 participants aged 10.5-15.5 years who performed the same test on two different days by two experienced observers. There were no significant differences between the test and retest $\mathrm{CSA}_{\mathrm{AT}}$ values. The $\mathrm{CV}$ was $2.6 \pm 1.2 \%$ and the RMSE was $2.1 \mathrm{~mm}^{2}$; a CV of $<5 \%$ is deemed acceptable.

\section{Calculation of AT Peak Stress, Strain and Young's Modulus}

Stress $(\sigma)$, determined as the force per unit area, was calculated using the minimum recorded $\mathrm{CSA}$ (i.e., $\mathrm{CSA}_{\mathrm{AT}}$ ) from each participant according to the equation: $\sigma=\mathrm{F} / \mathrm{A}$, where:

- F was the AT force $(\mathrm{N})$;

- A was the minimum $\mathrm{CSA}_{\mathrm{AT}}\left(\mathrm{mm}^{2}\right)$.
Tendon strain $(\varepsilon)$ was defined as tendon's elongation normalized to its test length according to the following equation: $\varepsilon$ $=\left(\Delta \mathrm{L} / \mathrm{L}_{\mathrm{o}}\right) \times 100 \%$ where $\mathrm{L}_{\mathrm{o}}$ and $\Delta \mathrm{L}$ represent the test length and elongation of the Achilles tendon, respectively. The 'peak strain' was not defined as the strain before failure of the tendon but the peak strain that could be induced by voluntary contraction (e.g., how much an individual might be able to induce strain by themselves). The Young's Modulus was calculated as the slope of stress $(\sigma)$ - strain $(\varepsilon)$ curve according to the equation: $\mathrm{E}=\mathrm{d} \sigma / \mathrm{d} \varepsilon$.

Over the 1.5 -year project period, standing height, sitting height, leg length, body mass, AT test length and minimum $\mathrm{CSA}_{\mathrm{AT}}$ were re-measured every 6 months. Achilles tendon moment arm, force, stiffness, Young's Modulus, strain and stress were calculated every 6 months. The maturation stage of 0 was defined as the age of PHV for all participants $(\mathrm{N}=$ 41). Table II shows combined data and percentage changes between successive maturation stages for tendon cross-sectional area, force, and tendon mechanical properties per stage of maturity.

Table II. Mean \pm SD values (combined data) and percentage changes between successive maturation stages (months from predicted age of PHV), for tendon cross-sectional area, force, and tendon mechanical properties per stage of maturity (months from predicted age of $\mathrm{PHV}$ ).

\begin{tabular}{|c|c|c|c|c|}
\hline Maturity stage & -12 & -6 & 0 & +6 \\
\hline $\mathrm{CSA}_{\mathrm{AT}}$ & $44.5 \pm 3$ & $\begin{array}{l}47 \pm 3 \\
(5.6 \%) \\
\end{array}$ & $\begin{array}{l}49 \pm 3 \\
(4.3 \%) \\
\end{array}$ & $\begin{array}{l}51.5 \pm 3 \\
(5.1 \%) \\
\end{array}$ \\
\hline $\mathrm{F}_{\text {peak }}$ & $1966.2 \pm 505,4$ & $2108.5 \pm 501,8(7.2 \%)$ & $2586.7 \pm 696.5^{* *}(22.7 \%)$ & $\begin{array}{l}2980 \pm 746.0 \\
(15.2 \%)\end{array}$ \\
\hline $\mathrm{k}_{\mathrm{low}}$ & $101.5 \pm 30.25$ & $\begin{array}{c}121.7 \pm 51.8 \\
(20.0 \%)\end{array}$ & $\begin{array}{l}142.5 \pm 62.2 \\
(17.1 \%)\end{array}$ & $\begin{array}{l}156.4 \pm 59 \\
(9.8 \%)\end{array}$ \\
\hline $\mathrm{E}_{\text {low }}$ & $338.3 \pm 105.85$ & $\begin{array}{c}409.5 \pm 178.2 \\
(21.0 \%) \\
\end{array}$ & $\begin{array}{l}494.1 \pm 212.0 \\
(20.7 \%) \\
\end{array}$ & $\begin{array}{l}530.5 \pm 205.7 \\
(7.3 \%)\end{array}$ \\
\hline$\varepsilon_{\text {low }}$ & $3.8 \pm 0.6$ & $\begin{array}{c}3.9 \pm 0.6 \\
(2.6 \%) \\
\end{array}$ & $\begin{array}{l}3.9 \pm 0.6 \\
(0.0 \%) \\
\end{array}$ & $\begin{array}{l}3.9 \pm 0.6 \\
(0.0 \%) \\
\end{array}$ \\
\hline$\sigma_{\text {low }}$ & $18.64 \pm 6.8$ & $\begin{array}{c}23.3 \pm 10.2 \\
(25.0 \%) \\
\end{array}$ & $\begin{array}{l}27.8 \pm 12.5 \\
(19.3 \%) \\
\end{array}$ & $\begin{array}{l}29.25 \pm 11.3 \\
(5.2 \%) \\
\end{array}$ \\
\hline $\mathrm{k}_{\text {lin }}$ & $199.5 \pm 47.3$ & $\begin{array}{l}311.9 \pm 151.7^{* \neq} \\
(56.3 \%)\end{array}$ & $\begin{array}{l}372.2 \pm 153.9 \\
(19.3 \%)\end{array}$ & $\begin{array}{l}448.3 \pm 165.6 \\
(20.4 \%)\end{array}$ \\
\hline $\mathrm{E}_{\text {lin }}$ & $664.0 \pm 162.5$ & $\begin{array}{l}1049.2 \pm 519.2^{* \neq} \\
(58.0 \%)\end{array}$ & $\begin{array}{l}1290.6 \pm 524.5^{5} \\
(23.0 \%)\end{array}$ & $\begin{array}{l}1519.0 \pm 574.1 \\
(17.7 \%)\end{array}$ \\
\hline$\varepsilon_{\text {peak }}$ & $\begin{array}{c}6.5 \pm 5.0 \\
(0.0 \%)\end{array}$ & $\begin{array}{l}6.8 \pm 1.1 \\
(4.6 \%)\end{array}$ & $\begin{array}{l}6.7 \pm 1.0 \\
(-1.5 \%)\end{array}$ & $\begin{array}{l}6.88 \pm 1.0 \\
(2.7 \%)\end{array}$ \\
\hline$\sigma_{\text {peak }}$ & $36.3 \pm 13.2$ & $\begin{array}{l}40.9 \pm 14.8 \\
(12.7 \%)\end{array}$ & $\begin{array}{l}45.8 \pm 15.4 \\
(12.0 \%)\end{array}$ & $\begin{array}{l}49.9 \pm 13.2 \\
(9.0 \%)\end{array}$ \\
\hline
\end{tabular}

CSA : minimum cross-sectional area $\left(\mathrm{mm}^{2}\right), \mathrm{F}_{\text {peak }}$ : peak force $(\mathrm{N}), \mathrm{k}$ : stiffness $(\mathrm{N} / \mathrm{mm})$, E: Young's modulus $(\mathrm{MPa}), \sigma:$ stress $(\mathrm{MPa}), \varepsilon:$ strain $(\%),($ low $)$ and (lin) indicate the low force level ( $10 \%-40 \%$ of peak force) and high force level (60\%-90\% of peak force; linear) region of force-elongation relationship. Significant change between consecutive stages of maturity in total sample: ${ }^{*}$ : $p<0.01$ total sample, ${ }^{\dagger}$ : $p<0.01$ within boys, ${ }^{*}$ : $p<0.01$ within girls. 


\section{Statistical analyses}

Maturation offset values were quantified to the nearest semester and grouped to integers such as - 12, - 6 months before and $0,+6$ months after predicted age of PHV in order to present the mean values (combined data) for AT dimensional and mechanical properties. Linear mixed models (LMM) were used to analyze inter-individual and intra-individual differences in changes in AT mechanical properties over maturity stages using SPSS statistical software (v.25.0, SPSS Inc., Chicago, USA). A diagonal covariance structure was used due to heterogeneous variances for each repeated measure and zero correlation between other repeated measures. An unstructured covariance structure was used as the most general covariance structure with no assumptions about the pattern of measurement errors within individuals. The estimation method of -2 Restricted Log Likelihood (-2RLL) was used as the main criterion for comparison with other models and according to the best fit of the model. Additionally, -2RLL was more accurate than Full Maximum Likelihood as the number of participants (N $=41)$ is small. Dependent variables (DVs) were peak force $\left(\mathrm{F}_{\text {peak }}\right)$, stiffness $\left(\mathrm{k}_{\text {low }}, \mathrm{k}_{\text {lin }}\right)$, Young's modulus $\left(\mathrm{E}_{\text {low }}, \mathrm{E}_{\text {lin }}\right)$, stress $\left(\sigma_{\text {low }}, \sigma_{\text {peak }}\right)$ and strain $\left(\varepsilon_{\text {low }}, \varepsilon_{\text {peak }}\right)$ in low $\left(10 \%-40 \%\right.$ of $\left.\mathrm{F}_{\text {peak }}\right)$ and high (60\%-90\% of $\mathrm{F}_{\text {peak }}$; linear region) force regions, respectively. Sex was used as a potential 2-level predictor (factor) for all dependent variables. Pairwise comparisons between two consecutive stages were conducted using Bonferroni confidence interval adjustment for each sex (tables I, II). Statistical significance was accepted at a $\mathrm{p}$-value $<0.05$.

\section{RESULTS}

Mean \pm SD values (combined data) for age, body mass and body height per stage of maturity are shown in table I. A significant change in height $(\mathrm{p}<0.01)$ was observed in the interval between 1 year before and the predicted age of PHV. Measured and calculated variables relating to AT mechanical properties are shown in table II.

\section{AT test length $\left(\mathrm{I}_{\text {test }}\right)$ and cross-sectional area $\left(\mathrm{CSA}_{A T}\right)$}

Analysis showed that $1_{\text {test }}$ and CSA $\mathrm{AT}_{\mathrm{TT}}$ were greater in boys than girls $(b=1.5395 \%$ CI [1.2-1.9], $\mathrm{p}<0.001 ; \mathrm{b}=4.90$ [4.18-5.57], $\mathrm{p}=0.023)$ although the rate of increase in $1_{\text {test }}$ and $\mathrm{CSA}_{\mathrm{AT}}(\mathrm{b}=0.05$ [- 1.4-0.3], $\mathrm{p}=0.594 ; \mathrm{b}=0.31[-$ $0.1-0.8], \mathrm{p}=0.176$ ) in boys and girls was similar. A significant increase in $1_{\text {test }}$ at PHV was observed in boys with limited increase thereafter (see table II). Increases in both $1_{\text {test }}(b=0.68[0.55-0.82], \mathrm{p}<0.001)$ and $\mathrm{CSA}_{\mathrm{AT}}(\mathrm{b}=1.55$
[1.23-1.87], $\mathrm{p}<0.001)$ were associated with maturation. No other significant increases in $1_{\text {test }}$ or $\mathrm{CSA}_{\mathrm{AT}}$ occurred between consecutive states of maturity in boys or girls (table II).

\section{AT Stiffness and Young's Modulus}

As shown in figure 3 , stiffness $\left(\mathrm{k}_{\mathrm{low}}\right)$ and Young's modulus $\left(\mathrm{E}_{\text {low }}\right)$ measured in the low-force region were greater in boys than girls $(b=44.18$ [24.03-64.32], $\mathrm{p}<0.001 ; \mathrm{b}=145.6$ [73.4-217.7], $\mathrm{p}<0.001$, respectively). $\mathrm{k}_{\text {low }}$ and $\mathrm{E}_{\text {low }}$ increased significantly with age relative to maturation offset $(b=9.63$ [4.21-15.04], $\mathrm{p}=0.001 ; \mathrm{b}=34.41$ [14.32-54.51], $\mathrm{p}=0.001$, respectively), but the rate of change over time (sex x maturation interaction) was not different between boys and girls $\left(\mathrm{p}=0.104 ; \mathrm{p}=0.118\right.$, respectively). $\mathrm{k}_{\text {low }}$ increased with force at $40 \%$ of $\mathrm{F}_{\text {peak }}(\mathrm{b}=0.07$ [0.03-0.11], $\mathrm{p}<0.001)$ but was not related to changes in $1_{\text {test }}, \mathrm{CSA}_{\mathrm{AT}}$, the ratio of $\mathrm{CSA}_{\mathrm{AT}}$ and $1_{\text {test }}$, or body mass. Thus, changes in $\mathrm{k}_{\text {low }}$ may be explained by changes in $\mathrm{E}_{\text {low }}$, as evidenced by its similar temporal response.

Both stiffness $\left(\mathrm{k}_{\text {lin }}\right)$ and Young's Modulus $\left(\mathrm{E}_{\text {lin }}\right)$ measured in the linear region was greater in boys than girls (see figure $3 ; b=88.90$ [38.56-139.25], $\mathrm{p}<0.001 ; \mathrm{b}=287.98$ [108.71467.25], $\mathrm{p}=0.002$, respectively). $\mathrm{k}_{\operatorname{lin}}$ and $\mathrm{E}_{\operatorname{lin}}$ increased significantly with maturation $(\mathrm{b}=39.97$ [23.95-52.00], $\mathrm{p}<$ $0.001 ; \mathrm{b}=132.67$ [81.85-183.48], $\mathrm{p}<0.001$, respectively), although the rates of increases in $\mathrm{k}_{\operatorname{lin}}$ and $\mathrm{E}_{\operatorname{lin}}$ were greater in boys than girls $(b=23.23$ [4.68-41.79], $\mathrm{p}=0.015 ; \mathrm{b}=80.49$ [12.97-148.01], $\mathrm{p}=0.020$, respectively). $\mathrm{k}_{\operatorname{lin}}$ increased with $\mathrm{F}_{\text {peak }}(\mathrm{b}=0.08$ [0.05-0.12], $\mathrm{p}<0.001)$, but the trend towards increase with body mass was not significant $(b=1.42[$ $0.13-3.00], \mathrm{p}=0.072) . \mathrm{k}_{\operatorname{lin}}$ also decreased with $1_{\text {test }}(\mathrm{b}=$ 14.07 [- 27.36-0.78], $\mathrm{p}=0.038$ ), but was not associated with changes in $\mathrm{CSA}_{\mathrm{AT}}$. Furthermore, $\mathrm{k}_{\text {lin }}$ was not related to the ratio between CSA $A_{A T}$ and $1_{\text {test }}$. Significant increases in $\mathrm{k}_{\operatorname{lin}}(\mathrm{p}$ $=0.002)$ and $\mathrm{E}_{\operatorname{lin}}(\mathrm{p}<0.001)$ were observed before PHV in boys while $\mathrm{E}_{\operatorname{lin}}$ increased significantly $(\mathrm{p}=0.044)$ at PHV in girls (table II). $\mathrm{E}_{\text {lin }}$ increased with body mass $(\mathrm{b}=5.95[0.47$ 11.42], $\mathrm{p}=0.034)$. The strain, as the ratio between elongation and $1_{\text {test }}$, was not different between the two groups $(\mathrm{p}$ $>0.05$ ). Mean strain difference was null between boys and girls at all stages of maturity, indicating that stiffness was calculated in similar stain sectors between sexes (figure 3).

\section{AT stress and AT strain}

Stress measured in both the low force $\left(\sigma_{\text {low }}\right.$; measured to $40 \%$ of $\left.\mathrm{F}_{\text {peak }} ; \mathrm{b}=7.93,[4.24-11.62], \mathrm{p}<0.001\right)$ and linear $\left(\sigma_{\text {peak }}\right.$; $\mathrm{b}=15.64$ [11.44-19.84], $\mathrm{p}<0.001)$ regions was greater in boys than girls. Increases in both $\sigma_{\text {low }}(b=2.27$ [1.10-3.43], $\mathrm{p}<0.001)$ and $\sigma_{\text {peak }}(\mathrm{b}=3.07$ [1.54-4.60], $\mathrm{p}<0.001)$ were 


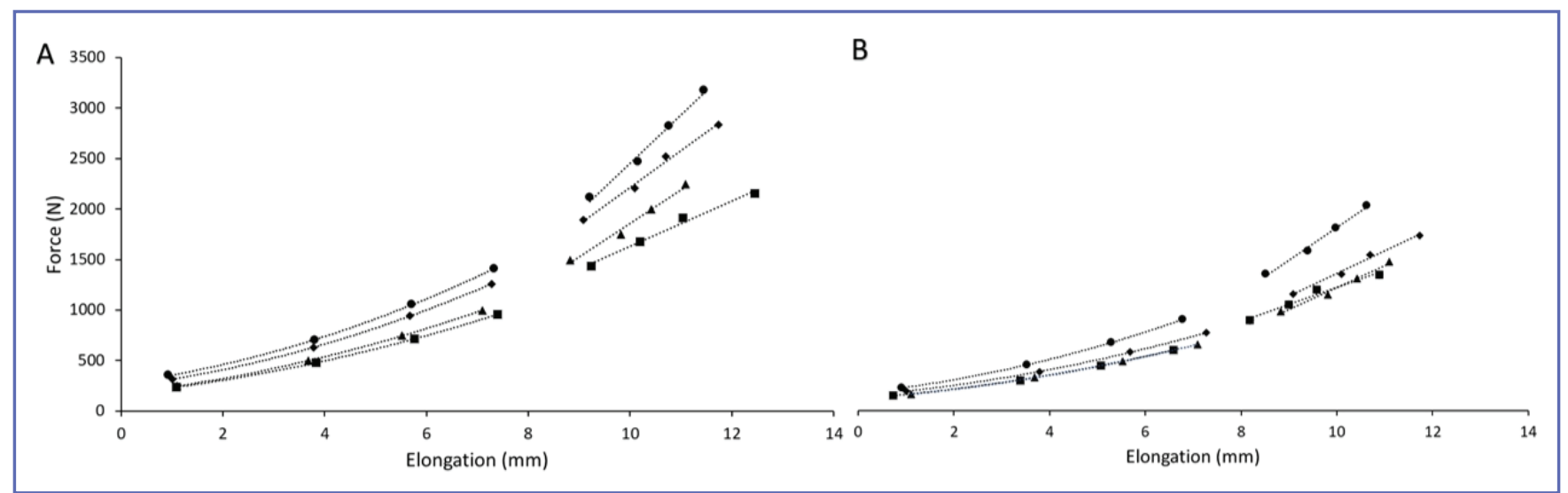

Figure 3. Mean force-elongation relationships in low force (i.e., 10\%-40\% of peak force) and linear (i.e., 60\%-90\% of peak force) regions per stage of maturity; - 12 (squares), - 6 (triangles), 0 (rhombuses), + 6 (circles), in boys $(A ; N=20)$ and girls ( $B ; N=21$ ). Boys showed greater increases in slope in both low force level and linear regions than girls, and greater increase between $3^{\text {rd }}$ and $4^{\text {th }}$ time point. The strain was not different among two groups $(p>0.05)$. Thus, stiffness was calculated in similar stain sectors.

associated with maturation, while the response between the sexes was similar and body mass had no effect. $\sigma_{\text {low }}$ also increased with increasing $\mathrm{k}_{\text {low }}$ and $\mathrm{E}_{\text {low }}(\mathrm{b}=0.19$ [0.17-0.20], $\mathrm{p}<0.001 ; \mathrm{b}=0.06$ [0.05-0.07], $\mathrm{p}<0.001$, respectively), and $\sigma_{\text {peak }}$ increased with increasing $\mathrm{k}_{\operatorname{lin}}$ and $\mathrm{E}_{\operatorname{lin}}(\mathrm{b}=0.18$ [0.010.03], $\mathrm{p}=0.005 ; \mathrm{b}=0.01$ [0.002-0.009], $\mathrm{p}=0.003$, respectively) when sex and $\mathrm{k}_{\operatorname{lin}}$ or $\mathrm{E}_{\operatorname{lin}}$ were entered as independent variables. As expected mathematically, $\sigma_{\text {peak }}$ was strongly related to $F_{\text {peak }}(\mathrm{p}<0.001)$. As shown in table II, $\varepsilon_{\text {low }}$ and $\varepsilon_{\text {peak }}$ were consistent across maturity stages as a result of parallel increases in length and tendon peak elongation.

\section{AT force}

Peak isometric plantar flexor force, and thus AT force $\left(\mathrm{F}_{\text {peak }}\right)$, was greater in boys than girls $(\mathrm{b}=879.38$ [697.501061.28], $\mathrm{p}<0.001$ ), and increases were associated with both maturation $(\mathrm{b}=147.95$ [80.21-215.70], $\mathrm{p}<0.001)$ and body mass $(b=9.86$ [2.07-17.64], $p=0.013)$. The response differed between sexes such that force increased more in boys than girls $(b=98.21[10.10-186.31], p=0.029) . F_{\text {peak }}$ showed a significant increase at PHV in boys $(\mathrm{p}<0.001)$ but increased relatively linearly in girls (table II).

\section{DISCUSSION}

The present longitudinal study describes changes in mechanical properties of an important energy-storing tendon, the Achilles tendon (AT), in boys and girls during pre-pubertal and adolescent developmental phases. Individuals were followed for 18 months (with testing at 0, 6, 12 and 18 months) and the data were combined to form a longitudinal data set for linear mixed model analysis. The results of the analysis, which are summarized in figure 4 , indicate that stiffness in both the low-force $\left(\mathrm{k}_{\text {low }}\right)$ and high-force (linear; $\left.\mathrm{k}_{\text {lin }}\right)$ regions of the force-length (stress-strain) relation was greater in boys than girls and increased similarly with age relative to peak height velocity (PHV), although boys showed a more prominent increase in $\mathrm{k}_{\operatorname{lin}}$ in the year before PHV. These changes in $\mathrm{k}_{\text {low }}$ and $\mathrm{k}_{\text {lin }}$ were most strongly related to changes in Young's modulus $\left(\mathrm{E}_{\text {low }}\right.$ and $\left.\mathrm{E}_{\text {lin }}\right)$ rather than AT cross-sectional area $\left(\mathrm{CSA}_{\mathrm{AT}}\right)$; stiffness and Young's moduli in both low force and linear regions were also greater in boys than girls but increased similarly with age except for the more notable increase in the year before PHV in boys. Similarly, force increased rapidly 6 months before PHV in boys. Therefore, changes in stiffness $k_{\text {low }}$ and $k_{\text {lin }}$ with age as well as differences between sexes were most strongly associated with changes/differences in maximal plantar flexor peak strength $\left(\mathrm{F}_{\text {peak }}\right)$, speculatively through a greater potential for force application to the tendon. Changes in $\mathrm{CSA}_{\mathrm{AT}}$ and tendon length $\left(\mathrm{l}_{\text {test }}\right)$ - or $\mathrm{CSA}_{\mathrm{AT}} / 1_{\text {test }}-$ did not change consistently with $\mathrm{F}_{\text {peak }}$, so age-related changes in tendon stress $\left(\sigma_{\text {low }}\right.$ and $\left.\sigma_{\text {peak }}\right)$ were strongly related to the changes in $\mathrm{F}_{\text {peak. }}$. However, since both muscular strength and AT stiffness increased simultaneously with age, the maximum tendon strain $\left(\varepsilon_{\text {peak }}\right)$ was constant across ages. Therefore, boys had greater AT stiffness than girls around PHV as a result of force changes rather than morphological changes. These results have important implications for our understanding of adaptation of energy-storing tendons during growth and maturation, and these are discussed below. 


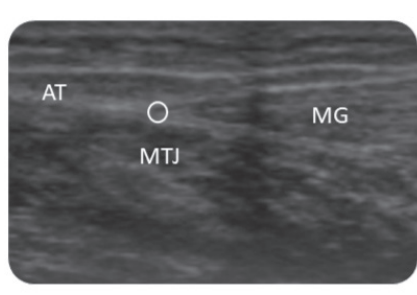

$10 \%-40 \%$ of $F_{\text {peak }}$

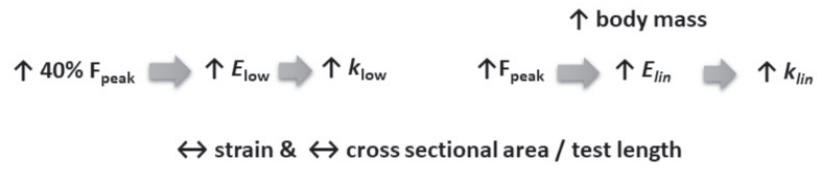

Body mass, $\mathrm{F}_{\text {peak }}, \sigma, E$ and $k$ were greater in boys than girls

Figure 4. Model of adaptation according to our longitudinal data. The increases in muscle force in the low force (10\%-40\% of $\left.F_{\text {peak }}\right)$, or force $\left(F_{\text {peak }}\right)$ and body mass in the linear region, were found to have the strongest relationship with changes in Young's modulus $\left(E_{\text {low' }}, E_{\text {lin }}\right)$ in both boys and girls before/ at PHV. Stiffness in low force $\left(k_{\text {low }}\right)$ and linear $\left(k_{\text {lin }}\right)$ regions were largely associated with changes in $\mathrm{E}_{\text {low }}$ and $\mathrm{E}_{\text {lin, }}$ respectively, indicating that changes in the tendon's material properties were key drivers of increases in stiffness. Body mass was only related to $\mathrm{E}_{\text {lin }}$. Strain and cross-sectional area/test length $1_{\text {test }}$ were unchanged. Therefore, force appears to drive Achilles tendon stiffness adaptation. Figure (top) shows longitudinal ultrasound images of the Achilles tendon (AT), in relaxed and contracted states. Myotendinous junction (MTJ) and medial gastrocnemius (MG) are also shown.

Previous cross-sectional data indicated that changes in Young's modulus (E) rather than tendon morphological properties underpinned changes in tendon stiffness during adolescent growth $(2,20)$. In the present study, both Achilles tendon stiffness $(\mathrm{k})$ and $\mathrm{E}$ increased similarly during the study time period (18 months) around the age of PHV, with high rates observed $\sim 1$ year before PHV; in fact, maturation, sex and the maturation-sex interaction were the common predictors of both $\mathrm{k}$ and $\mathrm{E}$ in linear region of the tendon force-length relation. These results are consistent with findings from cross-sectional study of Mogi, Torii (2), indicating that the peak rate of $\mathrm{k}$ increase is observed at or around PHV as a result of a greater rate of $\mathrm{E}$ increase during maturation. Increases in $\mathrm{k}$ are speculated to occur largely through adaptations including increased intrafibrillar crosslinking because of a lack of evidence of dimensional changes detected in cross-sectional cohorts $(2,3)$. Our data agree with these findings, showing that stiffness changes were unrelated to tendon cross-sectional area $\left(\mathrm{CSA}_{\mathrm{AT}}\right)$, length $\left(1_{\text {test }}\right)$ or the $\mathrm{CSA}_{\mathrm{AT}} / 1_{\text {test }}$ ratio.
Regarding to sex differences, linear mixed model analysis revealed that boys had greater $\mathrm{k}$ and $\mathrm{E}$ than girls in both force level regions. These results are consistent with several cross-sectional studies showing a lesser (patellar) tendon stiffness in young women than men $(21,22)$ but not consistent with others that observed no differences in AT between boys and girls cross-sectionally (3) or over a short (6 month) time period (6). One possibility is that our longitudinal analysis using LMM minimized the effects of between-subject variability, and thus statistical error, whilst allowing changes in AT to be tested over a period of several years. This analysis indicates that changes in muscle force production, with a small influence from body mass change, may underpin the observed sex differences.

The present analysis also leads to the assumption that the age-related increases in Achilles tendon $\mathrm{k}$ and $\mathrm{E}$ were likely predominately triggered by an increase in maximal plantar flexor force capacity $\left(\mathrm{F}_{\text {peak }}\right)$. This assumption is partly based on the findings that: 1 ) the rate of change in $\mathrm{F}_{\text {peak }}$ increased significantly just prior to PHV (table II), which followed the rapid increase in both $\mathrm{k}$ and $\mathrm{E}$ in boys and is also temporally aligned with the rapid increase in $\mathrm{E}$ in girls in the linear region; 2) robust statistical relationships were observed between $\mathrm{k}, \mathrm{F}_{\text {peak }}$ and $\mathrm{E}$; and 3) the significant association between force with maturation, body mass, sex and the interaction between sex and maturation, showing that force increased with maturation. However, boys produced greater force at all ages than girls and had correspondingly greater slopes of their force-, $\mathrm{k}$ - and E- maturation relations in both force level regions. These results might reflect a lesser adaptation before PHV in girls in particular, speculatively resulting from their lower physical activity levels (23). This is consistent with our findings that muscle strength and body mass stimuli for tendon adaptation, and therefore $\mathrm{E}$ measured in the linear region $\left(60 \%-90 \%\right.$ of $\left.\mathrm{F}_{\text {peak }}\right)$, was greater in boys and changed earlier than in girls. It would be of interest to compare adaptive changes in girls who perform a significant amount of physical activity (possibly including strength training) to those in the present study to determine whether adaptation might then be more similar between boys and girls.

Although tendon strain is commonly considered an important mechanical stimulus for tendon adaptation (9), our finding that $\varepsilon_{\text {peak }}(6.5-6.9 \%)$ was constant across maturation stages whilst $\sigma_{\text {low }}$ and $\sigma_{\text {peak }}$ were strongly related to $\mathrm{E}_{\text {low }}$ and $\mathrm{E}_{\text {lin }}$ (as well as $\mathrm{k}_{\text {low }}$ and $\mathrm{k}_{\text {lin }}$ ), respectively, is more consistent with the hypothesis that peak force, drives tendon stiffness adaptation. One possibility is that increases in tendon strain resulting from increases in muscle force production with maturation triggered rapid adaptations that subsequently limited tendon strain, i.e., strain was a predomi- 
nate trigger, which then restricted strain to constant values. The exact mechanism by which mechanical loading (force) might trigger adaptive change is not yet clear, however it might 1) result from tendon-specific molecular signaling in response to high forces imposed on local mechanosensitive molecules (24), or 2) relate to ubiquitous molecular signaling events in both the muscle and its associated connective tissues, including the tendons, triggered when high muscular forces are generated. By this latter mechanism, muscle (i.e., muscle strength) and tendon would undergo synergistic growth and adaptation during maturation in order to optimize muscle-tendon congruity (25). Thus, a link between muscle molecular signaling and tendon adaptation may exist. This concept is consistent with data showing a correlation between increases in muscle size and (patellar) tendon stiffness after a period of strength training in adult humans (26), as well as increases in both tendon CSA and stiffness in response to low-load (i.e., low tendon strain) blood flow-restricted exercise training leading to significant muscle hypertrophy (27). As the strategies for increasing tendon mass and altering mechanical function rely on a better understanding of the triggers for adaptation, molecular signaling and mechanical adaptation in energy-storing tendons may an important area for future study.

In this study there are some limitations. Firstly, the foot was accepted to be rigid. Thus, the effect of foot deformation on the changes in MTU length during contractions was considered negligible (28). In addition, there are no studies measuring the range of foot deformation in adolescents. Therefore, it was not investigated if foot deformation may cause incorrect results. Secondly, the ultrasound measurement may underestimate the $\mathrm{CSA}_{\mathrm{AT}}$ and the calculated stress may thus be overestimated. Previous research reported 5.5\% (29) smaller $\mathrm{CSA}_{\mathrm{AT}}$ measured with the ultrasound than the MRI

\section{REFERENCES}

1. Malina RM, Boushard C, Bar-Or O. Growth, maturation, and physical activity. (2 ed). Champain, IL USA: Human Kinetics 2004.

2. Mogi Y, Torii S, Kawakami Y, Yanai T. A cross-sectional study on the mechanical properties of the Achilles tendon with growth. Eur J Appl Physiol 2018;118(1):185-94.

3. Waugh CM, Blazevich AJ, Fath F, Korff T. Age-related changes in mechanical properties of the Achilles tendon. J Anatomy 2012;220(2):144-55.

4. Kubo K, Teshima T, Hirose N, Tsunoda N. Growth changes in morphological and mechanical properties of human patellar tendon in vivo. J Appl Biomech. 2014;30(3):415-22. method. Given that we measured the difference in Young's modulus between boys and girls in a longitudinally context, the methodological choice would not have strongly affected our finding that Young's modulus was greater between boys and girls at and/or around PHV. However, further analysis using MRI methods or $3 \mathrm{D}$ ultrasound may be needed to provide accurate $\mathrm{CSA}_{\mathrm{AT}}$ values.

\section{CONCLUSIONS}

In conclusion, Achilles tendon mechanical properties change throughout the adolescent growth period, although with a more rapid change before the age of peak height velocity in boys and with greater magnitude in boys than girls. The more rapid increase before PHV in boys was associated with an increase in force at that time, which may have evoked the molecular signaling required for adaptations to internal tendon structure. These mechanical changes were observed in both low- and high-force regions of the force-elongation relation. Our data are most consistent with a model in which increases in muscle force capacity (with a smaller effect of body mass, particularly for mechanical properties of the linear region of the force-elongation relation) trigger increases in modulus and thus stiffness. While both Achilles tendon CSA and length increase approximately linearly during adolescence, their changes appear to contribute little to changes in tendon stiffness. These data are the first to quantify longitudinal changes in the mechanical properties of an energy-storing, load-bearing tendon in humans as well as the factors that may influence them.

\section{CONFLICT OF INTERESTS}

The authors declare that they have no conflict of interests.

5. Kubo K, Kanehisa H, Fukunaga T. Gender differences in the viscoelastic properties of tendon structures. Eur J Appl Physiol 2003;88(6):520-6

6. Neugebauer JM, Hawkins DA. Identifying factors related to Achilles tendon stress, strain, and stiffness before and after 6 months of growth in youth 10-14 years of age. J Biomech 2012;45(14):2457-61.

7. Lefevre J, Beunen G, Steens G, Claessens A, Renson R. Motor performance during adolescence and age thirty as related to age at peak height velocity. Ann Hum Biol 1990;17(5):423-35.

8. Buchanan CI, Marsh RL. Effects of long-term exercise on the biomechanical properties of the Achilles tendon of guinea fowl. J Appl Physiol (1985) 2001;90(1):164-71. 
9. Arampatzis A, Peper A, Bierbaum S, Albracht K. Plasticity of human Achilles tendon mechanical and morphological properties in response to cyclic strain. J Biomech 2010;43(16):3073-9.

10. Mersmann F, Bohm S, Schroll A, Boeth H, Duda GN, Arampatzis A. Muscle and tendon adaptation in adolescent athletes: A longitudinal study. Scand J Med Sci Sports 2017;27(1):75-82.

11. Carmont MR, Brorsson, Karlsson J, Nilsson-Helander K. No difference in Achilles Tendon Resting Angle, Patient-reported outcome or Heel-rise height Index between Non-and Early-weightbearing the First Year after an Achilles Tendon Rupture. Muscles Ligaments Tendons J 2020;10(04):651-8.

12. Mirwald RL, Baxter-Jones AD, Bailey DA, Beunen GP. An assessment of maturity from anthropometric measurements. Med Sci Sports Exerc 2002;34(4):689-94.

13. Koo TK, Li MY. A Guideline of Selecting and Reporting Intraclass Correlation Coefficients for Reliability Research. J Chiropr Med 2016;15(2):155-63.

14. Padulo J, Oliva F, Frizziero A, Maffulli N. Basic principles and recommendations in clinical and field science research: 2018 update. Muscles Ligaments Tendons J 2018;8(3):305-7.

15. De Monte G, Arampatzis A, Stogiannari C, Karamanidis K. In vivo motion transmission in the inactive gastrocnemius medialis muscle-tendon unit during ankle and knee joint rotation. J Electromyogr Kinesiol 2006;16(5):413-22.

16. Arampatzis A, Monte GD, Karamanidis K. Effect of joint rotation correction when measuring elongation of the gastrocnemius medialis tendon and aponeurosis. J Electromyogr Kinesiol 2008;18(3):503-8.

17. Maganaris CN, Baltzopoulos V, Sargeant AJ. In vivo measurement-based estimations of the human Achilles tendon moment arm. Eur J Appl Physiol 2000;83(4 -5):363-9.

18. Mogi Y. The effects of growth on structural properties of the Achilles and Patellar tendons: A cross-sectional study. Physiol Rep 2020;8(16):e14544-e.

19. Bohm S, Mersmann F, Schroll A, Makitalo N, Arampatzis A. Insufficient accuracy of the ultrasound-based determination of Achilles tendon cross-sectional area. J Biomech 2016;49(13):2932-7.
20. Radnor JM, Oliver JL, Waugh CM, Myer GD, Moore IS, Lloyd RS. The Influence of Growth and Maturation on Stretch-Shortening Cycle Function in Youth. Sports Med 2018;48(1):57-71.

21. Westh E, Kongsgaard M, Bojsen-Moller J, et al. Effect of habitual exercise on the structural and mechanical properties of human tendon, in vivo, in men and women. Scand J Med Sci Sports 2008;18(1):23-30.

22. Furlong LAM, Harrison AJ. Sex-related differences in plantarflexor function during repeated stretch-shortening cycle loading. Muscles Ligaments Tendons J 2018;8(1):76-84.

23. Lima K, Martins N, Pereira W, Oliveira L. Triceps surae elasticity modulus measured by shear wave elastography is not correlated to the plantar flexion torque. Muscles Ligaments Tendons J 2017;7(2):347-52.

24. Kjaer M. Role of extracellular matrix in adaptation of tendon and skeletal muscle to mechanical loading. Physiol Rev 2004;84(2):649-98.

25. Gumucio JP, Sugg KB, Mendias CL. TGF-beta superfamily signaling in muscle and tendon adaptation to resistance exercise. Exerc Sport Sci Rev 2015;43(2):93-9.

26. Seynnes OR, Erskine RM, Maganaris CN, et al. Training-induced changes in structural and mechanical properties of the patellar tendon are related to muscle hypertrophy but not to strength gains. J Appl Physiol (1985) 2009;107(2):523-30.

27. Centner C, Lauber B, Seynnes OR, et al. Low-load blood flow restriction training induces similar morphological and mechanical Achilles tendon adaptations compared with high-load resistance training. J Appl Physiol (1985) 2019;127(6):1660-7.

28. Iwanuma S, Akagi R, Hashizume S, Kanehisa H, Yanai T, Kawakami Y. Triceps surae muscle-tendon unit length changes as a function of ankle joint angles and contraction levels: the effect of foot arch deformation. J Biomech 2011;44(14):2579-83.

29. Kruse A, Stafilidis S, Tilp M. Ultrasound and magnetic resonance imaging are not interchangeable to assess the Achilles tendon cross-sectional-area. Eur J Appl Physiol 2017;117(1):73-82. 